\title{
Moscas-das-frutas em pomares de pessegueiro e maracujazeiro, no Município de Iraceminha, Santa Catarina, Brasil
}

\author{
Fruit flies in peach and passion fruit orchards in Iraceminha, Santa Catarina, Brazil
}

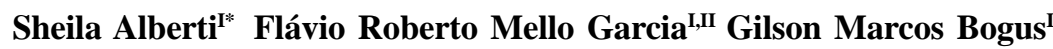

\begin{abstract}
Este estudo teve o objetivo de caracterizar a comunidade de moscas-das-frutas de ocorrência em pomares de pessegueiro e maracujazeiro, no Município de Iraceminha, Santa Catarina (SC), por meio da análise faunística. Os adultos de moscas-das-frutas foram capturados semanalmente, em armadilhas McPhail, contendo glicose invertida a 10\%, no período de abril de 2006 a março de 2007. Na caracterização, foram calculados os índices de abundância, constância, dominância, frequência e diversidade. Foram coletados 697 adultos de moscas-das-frutas. Anastrepha grandis e Anastrepha fraterculus foram as espécies mais abundantes, frequentes, constantes e dominantes nos pomares estudados, predominando sobre as outras espécies de moscas-das-frutas. Ceratitis capitata foi caracterizada como dispersa e pouco frequente. O maior índice de diversidade encontrado foi de 1,99 no pomar de maracujazeiro.
\end{abstract}

Palavras-chave: Anastrepha, diversidade, índice faunístico.

\section{ABSTRACT}

This research had the objective to characterize the community of fruit flies in peach and passion fruit orchards in Iraceminha, Santa Catarina, Brazil, through faunistic analyses. The adults fruit flies were weekly captured in McPhail traps with 10\% inverted glucose, from April 2006 to March 2007. In the characterization, the indices for abundance, constancy, dominance, frequency and diversity were calculated. A total of 697 adults fruit flies were collected. Anastrepha grandis and Anastrepha fraterculus species were the most abundant, frequent, constant and dominant in orchards studied, predominating upon other fruit flies species. Ceratitis capitata was dispersed and not very frequent. The biggest index of diversity of 1.99 was in passion fruit orchard.

Key words: Anastrepha, diversity, faunal index.

O Estado de Santa Catarina possui 70 espécies de moscas-das-frutas assinaladas (GARCIA et al., 2002). Esses insetos preocupam os produtores, pois causam aumento nos custos de produção, em razão das aplicações de inseticidas para seu controle, além de depreciarem a qualidade dos frutos e reduzirem o rendimento econômico. Estudos conduzidos em pomares comerciais mostraram que, embora diversas espécies de moscas-das-frutas estejam presentes, apenas uma ou duas são consideradas dominantes (URAMOTO et al., 2005).

Por meio da análise faunística, FERRARA et al. (2005) caracterizaram as populações de moscasdas-frutas em Itaperuna, região Noroeste do Estado do Rio de Janeiro, e concluíram que $\boldsymbol{A}$. fraterculus ocorreu com maior frequência (63,3\%), sendo a única espécie caracterizada como constante; a segunda espécie mais frequente foi $\boldsymbol{C}$. capitata (34,6\%), seguida por A. obliqua, sendo ambas caracterizadas como acessórias.

As espécies de moscas-das-frutas $\boldsymbol{A}$. fraterculus, A. obliqua e A. sororcula apresentaram

IPrograma de Pós-graduação em Ciências Ambientais, Universidade Comunitária Regional de Chapecó (UNOCHAPECÓ), Laboratório de Entomologia, CP 747, 89809-000, Chapecó, SC, Brasil. E-mail: sheilabio@unochapeco.edu.br. *Autor para correspondência. IIDepartamento de Zoologia e Genética, Instituto de Biologia, Universidade Federal de Pelotas (UFPel), Pelotas, RS, Brasil. 
os maiores índices de frequência, constância e dominância, em estudo realizado nos cinco municípios do Estado do Rio de Janeiro (AGUIAR-MENEZES et al., 2008). O presente estudo teve por objetivos registrar a diversidade de espécies de moscas-das-frutas (Diptera: Tephritidae) de ocorrência em pomares de pessegueiro e maracujazeiro, no Município de Iraceminha, Santa Catarina (SC), e caracterizar suas populações por meio da análise faunística.

As coletas de moscas-das-frutas foram realizadas de abril de 2006 a março de 2007, no Município de Iraceminha, região Oeste de Santa Catarina, em três pomares: pomar de maracujazeiro, localizado em área rural a 50m do pomar de pessegueiro “ $\mathrm{A}$ ”, com predomínio de produção agrícola diversificada de legumes e pequena área de mata nativa nas proximidades; e pomar de pessegueiro “B”, com vegetação nativa e pomar de citros aos arredores, localizado a uma distância aproximada de $3 \mathrm{~km}$ dos demais pomares. O pessegueiro teve sua produção iniciada em 1995, e o maracujazeiro foi implantado no ano de 2003, em propriedade rural do município. Os produtores realizavam tratamento fitossanitário, tanto no pessegueiro, quanto no maracujazeiro. A idade das plantas do pessegueiro variava de 13 a 15 anos e do maracujazeiro variava de um a três anos.

Para coleta de adultos, foram instaladas armadilhas do tipo McPhail, contendo 200ml de solução aquosa de glicose invertida a 10\% (GARCIA et al., 1999). Foi utilizado um total de 20 armadilhas, sempre distribuídas nas bordas dos pomares. Foram realizadas visitas semanais aos pomares para a troca da solução atrativa e coleta dos insetos, totalizando 52 coletas. Os insetos capturados foram conservados em álcool 70\%, para posterior separação por sexo, contagem e identificação específica. A identificação das espécies de Anastrepha foi baseada em STEYSKAL (1977) e ZUCCHI (2000), e os outros gêneros foram identificados por meio das chaves confeccionadas por FOOTE(1980).

As populações de moscas-das-frutas foram caracterizadas por meio da análise faunística, determinando os índices de frequência, constância, abundância, dominância e diversidade de espécies. Cada pomar foi considerado como tendo uma comunidade de moscas-das-frutas com características próprias. A frequência foi determinada por meio da porcentagem de indivíduos de cada espécie, em relação ao total de adultos de moscas-das-frutas obtidos nas armadilhas em cada pomar, e classificadas nas seguintes categorias: pouco frequentes, frequentes ou muito frequentes (THOMAZINI \& THOMAZINI, 2002). A constância foi determinada para cada espécie pela equação citada por SILVEIRA NETO et al. (1976) $\mathrm{C}=\mathrm{px} 100 / \mathrm{N}$, em que $\mathrm{p}$ : número de coletas contendo a espécie e $\mathrm{N}$ : número total de coletas, sendo caracterizadas nas seguintes categorias: constante, acessória ou acidental.

Os cálculos e classificações (rara, dispersa, comum, abundante e muito abundante) da abundância foram realizados de acordo com GARCIA \& CORSEUIL (1998). Quanto à dominância, as espécies foram classificadas em dominantes quando os valores de frequência foram superiores ao limite calculado pela equação citada por SILVA (1993) LD=1/Sx100, em que S: número total de espécies. Foi também calculado o índice de diversidade para cada pomar, por meio da equação proposta por Margalef (1951), citada por SILVEIRA NETO et al. (1976), $\alpha=$ s-1/Log N, em que s: número de espécies amostradas e $\mathrm{N}$ : número total de indivíduos em todas as espécies.

No período de amostragem, foram capturados 697 exemplares de moscas-das-frutas de 12 espécies pertencentes a quatro gêneros: Anastrepha barbiellinii Lima, Anastrepha dissimilis Stone, Anastrepha fraterculus Wiedemann, Anastrepha grandis Macquart, Anastrepha montei Lima, Anastrepha obliqua Macquart, Anastrepha pseudoparallela Loew, Anastrepha sororcula Zucchi, Anastrepha xanthochaeta Hendel, Blepharoneura Loew, Ceratitis capitata Wiedemann, e Tomoplagia Coquillett.

A. grandis foi a espécie mais abundante, mais frequente, constante e dominante no pomar de maracujazeiro (Tabela 1). Segundo MALAVASI et al. (2000), A. grandis tem sua importância relacionada aos aspectos quarentenários da produção de melão e sua ocorrência no pomar estudado pode ter ocorrido pelo fato de serem atraídas pela quantidade de cucurbitáceas que se encontrava nas proximidades do pomar, sendo um dos seus principais hospedeiros.

As espécies $\boldsymbol{A}$. barbiellinii, $\boldsymbol{A}$. montei, $\boldsymbol{A}$. obliqua, A. pseudoparallela, A. sororcula, A. xanthochaeta, Blepharoneura sp. e $C$. capitata foram acidentais, provavelmente porque poucos exemplares foram capturados e em apenas algumas semanas. Essas espécies apresentaram índices muito baixos. $\boldsymbol{A}$. fraterculus foi a segunda espécie mais coletada. A coleta dessas moscas pode ter ocorrido devido à presença de plantas hospedeiras que se encontravam muito próximas do maracujazeiro. AGUIAR-MENEZES et al. (2008) concluíram que em São Francisco do Itabapona, Estado do Rio de Janeiro, A. fraterculus foi a segunda espécie mais frequente $(15,8 \%)$.

O maior índice de diversidade encontrado foi de 1,99, representado pelas 10 espécies ocorrentes 
Tabela 1 - Análise faunística de moscas-das-frutas coletadas com “caça moscas” do tipo McPhail em pomares de maracujazeiro, pessegueiro “A” e pessegueiro “B”, no Município de Iraceminha, oeste catarinense, no período de abril de 2006 a março de 2007.

\begin{tabular}{|c|c|c|c|c|c|}
\hline Espécies & Pomares & Abundância & Frequência & Constância & Dominância \\
\hline A. barbiellinii & Maracujazeiro & Comum & Frequente & Acidental & Dominante \\
\hline A. barbiellinii & Pessegueiro “A”, pessegueiro “B” & Rara & Pouco frequente & Acidental & Não-dominante \\
\hline A. dissimilis & Pessegueiro “B” & Rara & Pouco frequente & Acidental & Não-dominante \\
\hline A. fraterculus & Maracujazeiro, pessegueiro “B” & $\begin{array}{l}\text { Muito } \\
\text { Abundante }\end{array}$ & Muito frequente & Acessória & Dominante \\
\hline A. fraterculus & Pessegueiro “A” & Muito abundante & Muito frequente & Constante & Dominante \\
\hline A. grandis & Maracujazeiro, pessegueiro “B” & Muito abundante & Muito frequente & Constante & Dominante \\
\hline A. grandis & Pessegueiro “A” & Comum & Frequente & Constante & Dominante \\
\hline A. montei & Maracujazeiro & Rara & Pouco frequente & Acidental & Não-dominante \\
\hline A. oblíqua & Maracujazeiro & Rara & Pouco frequente & Acidental & Não-dominante \\
\hline A. pseudoparallela & Maracujazeiro, pessegueiro “A” e "B" & Rara & Pouco frequente & Acidental & Não-dominante \\
\hline A. sororcula & Maracujazeiro, pessegueiro “A” e "B” & Rara & Pouco frequente & Acidental & Não-dominante \\
\hline A. xanthochaeta & Maracujazeiro & Rara & Pouco frequente & Acidental & Não-dominante \\
\hline Blepharoneura sp. & Maracujazeiro, pessegueiro “A” e "B” & Rara & Pouco frequente & Acidental & Não-dominante \\
\hline Ceratitis capitata & Maracujazeiro, pessegueiro “A” & Rara & Pouco frequente & Acidental & Não-dominante \\
\hline Ceratitis capitata & Pessegueiro “B” & Dispersa & Pouco frequente & Acidental & Não-dominante \\
\hline Tomoplagia sp. & Pessegueiro “A” & Rara & Pouco frequente & Acidental & Não-dominante \\
\hline Índice de diversidade & Maracujazeiro & 1,99 & & & \\
\hline Índice de diversidade & Pessegueiro “A” & 1,43 & & & \\
\hline Índice de diversidade & Pessegueiro “B” & 1,42 & & & \\
\hline
\end{tabular}

no pomar de maracujazeiro. Esse valor chega próximo ao índice encontrado por GARCIA et al. (2003) no Município de Chapecó - SC $(2,0)$. No pomar de pessegueiro “A”, pelos valores dos índices da análise faunística contidos na tabela $1, \boldsymbol{A}$. fraterculus foi a espécie mais abundante, considerada como predominante no pomar estudado, seguida por $\boldsymbol{A}$. grandis. A abundância de $\boldsymbol{A}$. fraterculus deve-se ao fato de o pêssego ser uma das suas principais plantas hospedeiras. Resultado semelhante foi obtido por GARCIA et al. (2003), em que A. fraterculus foi muito abundante em todos os pomares, nos estudos realizados nos Municípios da região Oeste de Santa Catarina. Os baixos índices de constância obtidos para A. barbiellinii, A. pseudoparallela, A. sororcula e Blepharoneura sp. sugerem que a ocorrência dessas espécies no pomar de pessegueiro “A” seja considerada esporádica. C. capitata, mesmo sendo uma importante praga do pêssego, não teve ocorrência dominante nesse pomar. Apenas um exemplar de Tomoplagia sp. foi coletado, sua ocorrência foi rara no levantamento realizado no Município de Iraceminha, portanto, não sendo de expressão nesse local.

O índice de diversidade do pomar de pessegueiro "A" ficou em 1,43. Esse valor tende a ser baixo em local onde os fatores limitantes e a competição interespecífica atuam intensamente. Esse valor é maior que o índice encontrado por GARCIA \& LARA (2006), em estudo no Município de Dionísio Cerqueira - SC, onde as espécies de moscas-das-frutas amostradas correspondem ao índice de diversidade de 1,09.

No pomar de pessegueiro " $B$ ", com base nos valores observados de abundância, frequência e dominância de A. fraterculus e A. grandis (Tabela 1), sugere-se que essas duas espécies demonstram seu maior sucesso competitivo em comparação às demais espécies, que foram raras (exceto Ceratitis capitata) e não-dominantes. Esses resultados discordam do que foi observado por GARCIA et al. (2003), em que $\boldsymbol{A}$. fraterculus foi dominante em todas as culturas estudadas, em quatro Municípios da região Oeste de Santa Catarina (Chapecó, Cunha Porã, São Carlos e Xanxerê); enquanto $\boldsymbol{A}$. grandis somente foi dominante nos dois pomares de Xanxerê.

Foram poucos os exemplares dos gêneros Ceratitis e Blepharoneura sp. obtidos nas armadilhas, pois a maioria das espécies obtidas foram do gênero Anastrepha. Esse resultado também foi obtido por BOMFIM et al. (2007), em que Anastrepha foi o gênero com maior percentagem de adultos capturados pelas armadilhas McPhail (74,5\%). O menor índice de diversidade foi obtido no pomar de pessegueiro " $B$ " $(1,42)$, sendo que as espécies mais comuns tendem a aumentar suas populações, e as espécies raras a apresentar baixo nível populacional. Nos três locais estudados, os índices foram baixos e não muito diferentes entre si.

Ciência Rural, v.39, n.5, ago, 2009. 
Verificou-se que as espécies não foram muito variáveis de um local para outro, porém os índices das espécies predominantes sempre foram muito altos, enquanto as não-dominantes foram representadas apenas por alguns exemplares. A. fraterculus e $\boldsymbol{A}$. grandis foram as principais espécies capturadas no levantamento realizado.

\section{REFERÊNCIAS}

AGUIAR-MENEZES, E.L. et al. Análise faunística de moscasdas-frutas (Diptera: Tephritidae) nas regiões norte e noroeste do Estado do Rio de Janeiro. Neotropical Entomology, v.37, n.1, p.8-14, 2008. Disponível em: <http://www.scielo.br/ scielo.php? script =sci_art text \& pid = S 1519 $566 \times 2008000100002 \& \operatorname{lng}=p t \& n r m=i s o>$. Doi: $10.1590 /$ S1519-566X2008000100002.

BOMFIM, D.A. do et al. Biodiversidade de moscas-das-frutas (Diptera, Tephritoidea) em matas nativas e pomares domésticos de dois municípios do Estado do Tocantins, Brasil. Revista Brasileira de Entomologia, v.51, n.2, p.217-223, 2007. Disponível em: $<$ http://www.scielo.br/scielo.php?script=sci_arttext\&pid=S008556262007000200012\&lng=pt\&nrm=iso > . Doi: 10.1590/S008556262007000200012 .

FERRARA, F.A.A. et al. Análise faunística de moscas-dasfutas (Diptera: Tephritidae) da região noroeste do estado do Rio de Janeiro. Neotropical Entomology, v.34, n.2, p.183190, 2005. Disponível em: <http://www.scielo.br/ scielo.php ? script=sci_art text \& pid = S 1519 566X2005000200006\&lng=pt\&nrm=iso>. Doi: $10.1590 /$ S1519-566X2005000200006.

FOOTE, R.H. Fruit fly genera south of the United States (Diptera: Tephritidae). Washington D.C.: Science Education Administration, 1980. 79p.

GARCIA, F.R.M.; CORSEUIL, E. Análise faunística de moscasdas-frutas (Diptera, Tephritidae) em pomares de pessegueiro em Porto Alegre, Rio Grande do Sul. Revista Brasileira de Zoologia, v.15, n.4, p.1111-1117, 1998.

GARCIA, F.R.M.; LARA, D.B. de. Análise faunística e flutuação populacional de moscas-das-frutas (Diptera, Tephritidae) em pomar cítrico no município de Dionísio Cerqueira, Santa Catarina. Biotemas, v.19, n.3, p.65-70, 2006.

GARCIA, F.R.M. et al. Avaliação de atrativos na captura de adultos de Anastrepha fraterculus (Wied., 1830) (Diptera, Tephritidae). Biociências, v.7, n.1, p.43-50, 1999.
GARCIA, F.R.M. et al. Lista documentada das moscas-dasfrutas (Diptera, Tephritidae) de Santa Catarina, Brasil. Biociências, v.10, n.1, p.139-148, 2002.

GARCIA, F.R.M. et al. Análise faunística de espécies de moscas-dasfrutas (Diptera: Tephritidae) na região Oeste de Santa Catarina. Neotropical Entomology, v.32, n.3, p.421-426, 2003. Disponível em: <http://www.scielo.br/scielo.php?script=sci_arttext\&pid=S1519566X2003000300006\&lng=pt\&nrm=iso > . Doi: 10.1590/S1519566X2003000300006.

MALAVASI, A. et al. Biogeografia. In: MALAVASI, A.; ZUCCHI, R.A. (Eds.). Moscas-das-frutas de importância econômica no Brasil: conhecimento básico e aplicado. Ribeirão Preto: Holos, 2000. 327p. p.93-98.

SILVA, N.M. da. Levantamento e análise faunística de moscas-das-frutas (Diptera: Tephritidae) em quatro locais do Estado do Amazonas. 1993. 152f. Tese (Doutorado em Entomologia) - ESALQ, Universidade de São Paulo, Piracicaba, SP.

SILVEIRA NETO, S. et al. Manual de ecologia dos insetos. Piracicaba: Agronômica Ceres, 1976. 419p.

STEYSKAL, G.C. Pictorial key to species of the genus Anastrepha (Diptera: Tephritidae). Washington: The Entomological Society of Washington, 1977. 35p.

THOMAZINI, M.J.; THOMAZINI, A.P. de B.W. Diversidade de abelhas (Hymenoptera: Apoidea) em inflorescências de Piper hispidinervum (C.DC.). Neotropical Entomology, v.31, n.1, p.27-34, 2002. Disponível em: <http://www.scielo.br/ scielo.php? script=sci_arttext\&pid=S 1519 566X2002000100004\&lng=pt\&nrm=iso>. Doi: 10.1590/ S1519-566X2002000100004.

URAMOTO, K. et al. Análise quantitativa e distribuição de populações de espécies de Anastrepha (Diptera: Tephritidae) no campus Luiz de Queiroz, Piracicaba, SP. Neotropical Entomology, v.34, n.1, p.33-39, 2005. Disponível em: <http:/ /www.scielo.br/scielo.php?script=sci_arttext\&pid=S1519$566 \times 2005000100005 \& \operatorname{lng}=$ pt $\&$ nrm=iso $>$. Doi: 10.1590/ S1519-566X2005000100005.

ZUCCHI, R.A. Taxonomia. In: MALAVASI, A.; ZUCCHI, R.A. (Eds.). Moscas-das-frutas de importância econômica no Brasil: conhecimento básico e aplicado. Ribeirão Preto: Holos, 2000. 327p. p.13-24. 